\title{
Downregulation of high mobility group box 1 enhances the radiosensitivity of non-small cell lung cancer by acting as a crucial target of microRNA-107
}

\author{
LU BAI, JINGJING ZHANG, DONGQI GAO, CHENGYI LIU, WENXIN LI and QINGSHAN LI
}

\author{
Department of Oncology, Affiliated Hospital of Chengde Medical University, Chengde, Hebei 067000, P.R. China
}

Received April 28, 2020; Accepted April 9, 2021

DOI: $10.3892 / \mathrm{etm} .2021 .10111$

\begin{abstract}
High mobility group box 1 (HMGB1) has been reported to regulate the sensitivity of several types of cancer cell to chemoradiotherapy. The present study aimed to investigate the changes in HMGB1 expression after radiotherapy, as well as its regulatory role in the radiosensitivity of non-small cell lung cancer (NSCLC) cells. The expression levels of HMGB1 in the serum of 73 patients with NSCLC were analyzed by ELISA. HMGB1 mRNA and microRNA (miR)-107 expression in NSCLC cells were assessed using reverse transcription-quantitative PCR. Receiver operating characteristic analysis was used to evaluate the diagnostic value of HMGB1. Cell counting kit-8, Transwell invasion and clonogenic assays were used to determine cellular viability, invasiveness and colony formation ability, respectively. Following radiotherapy, the levels of HMGB1 were significantly decreased in the serum of patients with NSCLC, and lower serum levels had relatively high diagnostic accuracy in radiosensitive patients. Furthermore, HMGB1-knockdown retarded cellular proliferation and invasion with or without irradiation, and enhanced NSCLC cell radiosensitivity. Furthermore, knocking down miR-107 reversed the decreases in cellular proliferation and invasiveness both with and without irradiation, and reduced the survival fractions induced by sh-HMGB1.HMGB1-knockdown leads to radiosensitivity that may result from suppression of the Toll-like receptor 4 (TLR4)/NF- $\mathrm{kB}$ signaling pathway. Collectively, decreased expression of HMGB1 was found to be a putative diagnostic predictor of radiosensitivity in patients with NSCLC. HMGB1-knockdown inhibited the proliferation and enhanced the radiosensitivity of NSCLC cells, which may be regulated via miR-107 by mediating the TLR4/NF- $\mathrm{BB}$ signaling pathway. Thus, HMGB1 may be a potential regulator
\end{abstract}

Correspondence to: Dr Qingshan Li, Department of Oncology, Affiliated Hospital of Chengde Medical University, Daliu Line Road, Brick and Tile Kiln Area, Fengyingzi Town, High-tech Industrial Development Zone, Chengde, Hebei 067000, P.R. China

E-mail: qingshanli036@163.com

Key words: diagnosis, high mobility group box 1, non-small cell lung cancer, radiosensitivity of radioresistance in NSCLC, and the HMGB1/miR-107 axis may represent a promising therapeutic target.

\section{Introduction}

Lung cancer is the most common malignant tumor with the highest morbidity and mortality rates, and is one of the most life-threatening cancers worldwide (1). Based on histological type, lung cancer is classified as small cell lung cancer and non-small cell lung cancer (NSCLC); of these subtypes, NSCLC accounts for $\sim 80-85 \%$ of all lung cancer cases (2). At present, the effects of NSCLC treatment are not satisfactory, and no major breakthrough in survival outcome has been achieved. In addition, most patients are in the advanced stages of disease at initial diagnosis, have missed the opportunity for surgery, and cannot tolerate systemic chemotherapy; thus, radiotherapy has become an alternative method for the treatment of NSCLC (3). However, recurrence and distant metastasis remain major obstacles in the implementation of this therapeutic method. Thus, it is essential to identify specific molecular markers to predict the response to radiotherapy in patients with invasive disease; such markers may increase the efficacy of radiotherapy for NSCLC.

High mobility group box 1 (HMGB1), located in the eukaryotic nucleus, is a nonhistone DNA-binding protein involved in various cellular functions, such as inflammation, proliferation, differentiation and metastasis (4-6). A number of studies have demonstrated that as a chromatin-binding nuclear protein, HMGB1 is highly expressed in multiple tumor types, which is correlated with tumor development and progression $(6,7)$. The expression levels of HMGB1 have also been associated with the chemosensitivity or radiosensitivity of several types of cancer (8-10). In lung cancer, HMGB1 expression is upregulated, promotes tumor cell proliferation, and is associated with tumor cell chemosensitivity (11-13). However, the function of HMGB1 in the radioresistance of NSCLC, as well as the underlying mechanism, remains unclear.

MicroRNAs (miRNAs) are a group of small endogenous noncoding RNA molecules that downregulate gene expression by repressing or degrading their target mRNAs. miRNAs are implicated in various pathological and physiological processes through the regulation of a wide array of cellular functions, such as differentiation, apoptosis, proliferation and metastasis $(14,15)$. Numerous studies have indicated that the abnormal expression of specific miRNAs may be involved in the acquisition 
of radioresistance in numerous cancer types, particularly NSCLC (16-18). miR-107 is aberrantly expressed in various malignancies and can function as a tumor suppressor or oncogene by regulating cellular proliferation and invasiveness. In NSCLC, miR-107 inhibits tumor growth and metastasis by regulating the brain-derived neurotrophic factor (BDNF)-mediated PI3K/AKT pathway, demonstrating that the miR-107/BDNF axis may contribute to NSCLC tumorigenesis (19). Additionally, targeting CDK8 via miR-107 may promote the reversal of chemoresistance, and provide a biomarker for cisplatin responsiveness in patients with NSCLC (20). However, the level of miR-107 in the serum of patients with NSCLC, and its role in radiotherapy, require further investigation.

In the present study, the expression levels of HMGB1 were detected in patients with NSCLC who had received radiotherapy, and its diagnostic value for distinguishing radiotherapy-sensitive from radiotherapy-resistant patients was analyzed. Furthermore, the effect of HMGB1 on the radiosensitivity of A549 and H1299 cells was investigated in vitro. Specifically, a direct interaction between miR-107 and the 3' untranslated region (UTR) of HMGB1 was observed, suggesting a novel mechanism by which HMGB1 and miR-107 regulate NSCLC cell proliferation and radiosensitivity.

\section{Materials and methods}

Patients and serum samples. The present study was approved by the Ethics Committee of the Affiliated Hospital of Chengde Medical University, and all the patients provided written informed consent. A total of 73 patients ( 31 female and 42 male; mean age, 55.24 years; age range, 26-75 years) who were pathologically diagnosed with NSCLC were enrolled into the present study between January 2017 and December 2018, among. The patients were recruited based on the following inclusion criteria: i) Aged $<70$ years old, and with NSCLC, confirmed by histopathology; ii) had an expected survival time $>3$ months; iii) had received chest radiotherapy for the first time, without concurrent chemotherapy or immunomodulator treatment; iv) had a Karnofsky performance score $>70$; and v) exhibited normal heart, liver and kidney function. All patients received radiotherapy with intensity-modulated radiation therapy on an Elekta Synergy linear accelerator, and the total radiation dose was $60 \mathrm{~Gy}$ ( 2 fractions at $30 \mathrm{~Gy}$ ).

Fasting venous blood was collected from the patients 1 week prior to, and 2, 4 and 6 weeks after radiotherapy. The blood samples were centrifuged at $700 \mathrm{xg}$ for $10 \mathrm{~min}$ at $-4^{\circ} \mathrm{C}$ to isolate the serum, which was then stored at $-80^{\circ} \mathrm{C}$ for subsequent analysis.

ELISA. The HMGB1 concentrations in the blood samples were determined using an HMGB1 ELISA kit (cat. no. ARG81351; arigo Biolaboratories Corp.) according to the manufacturer's instructions.

Cell culture and X-ray irradiation. The NSCLC cell lines, A549 and H1299, were obtained from the Shanghai Institute of Cell Biochemistry and Cell Biology (Shanghai, China). The cells were cultured in DMEM supplemented with $10 \%$ FBS (both Invitrogen; Thermo Fisher Scientific, Inc.) at $37^{\circ} \mathrm{C}$ and a $\mathrm{CO}_{2}$ concentration of $5 \%$.
NSCLC cell irradiation was conducted at room temperature using a 6-MV linear accelerator (Siemens AG), with a source skin distance of $100 \mathrm{~cm}$ and an irradiation dose rate of $200 \mathrm{cGy} / \mathrm{min}$.

Transfection. The miR-107 mimics (5'-AGCAGCAUUGUACA GGGCUAUCA-3'), mimic negative control (NC; 5'-UUCUCC GAACGUGUCACGUTT-3'), miR-107 inhibitor (5'-UGAUAG CCCUGUACAAUGCUGCU-3') and inhibitor NC (5'-CAGUA CUUUUGUGUAGUACA-3') were synthesized and purchased from Shanghai GenePharma Co., Ltd. Preliminary experiments were carried out using A549 and H1299 cells transfected with $50 \mathrm{nM}$ miR-107 mimics, inhibitor or the relevant NCs.

To knockdown HMGB1, shRNA HMGB1 or a scrambled shRNA sequence was sub-cloned into pGPU6/Neo plasmids (Shanghai GenePharma Co., Ltd.), referred to as sh-HMGB1 or scrambled control. A549 and H1299 cells were transfected with sh-HMGB1 or the associated scrambled control.

All transfections were performed using Lipofectamine ${ }^{\circledR} 2000$ (Invitrogen; Thermo Fisher Scientific, Inc.) according to the manufacturer's protocol. After transfection for $24 \mathrm{~h}$ at $37^{\circ} \mathrm{C}$, the transfection efficiency was verified using reverse transcription-quantitative PCR (RT-qPCR).

$R N A$ extraction and $R T-q P C R$. Total RNA was extracted from blood using the miRNeasy Mini Kit (Qiagen $\mathrm{GmbH}$ ), and from NSCLC cells using TRIzol ${ }^{\circledR}$ reagent (Invitrogen; Thermo Fisher Scientific, Inc.) per the manufacturers' instructions. Then, the total RNA was reverse transcribed into cDNA using the miScript II RT Kit (Qiagen $\mathrm{GmbH}$ ) or PrimeScript $^{\mathrm{TM}}$ RT Master Mix (Takara Bio, Inc.) according to the manufacturers' instructions. qPCR was performed using the SYBR Premix Ex Taq II PCR kit (Takara Bio, Inc.) according to the manufacturer's protocol. The thermocycling conditions were as follows: $95^{\circ} \mathrm{C}$ for $5 \mathrm{~min}$, followed by 35 cycles of $95^{\circ} \mathrm{C}$ for $20 \mathrm{sec}, 60^{\circ} \mathrm{C}$ for $30 \mathrm{sec}$, and $72^{\circ} \mathrm{C}$ for $20 \mathrm{sec}$, with a final extension at $72^{\circ} \mathrm{C}$ for $5 \mathrm{~min}$. The relative levels of miR-107 and HMGB1 expression were calculated using the $2^{-\Delta \Delta C q}$ method (21), and U6 and GAPDH served as the internal controls for miRNA and mRNA expression, respectively. The qPCR primer sequences were as follows: miR-107 forward, 5'-GCCGAGAGCAGCAUUGUACA-3', and reverse, 5'-CTCAACTGGTGTCGTGGA-3'; HMGB1 forward, 5'-CCA GGGGCTTTTCTACCAGG-3' and reverse, 5'-AACCCCAAC AAACTGCTGGA-3';U6forward,5'-GTGCTCGCTTCGGCA GCACATATAC-3' and reverse, 5'-AAAAATATGGAACGC TCACGAATTTG-3'; and GAPDH forward, 5'-ACCCAGAAG ACTGTGGATGG-3' and reverse, 5'-CACATTGGGGGTAG GAACAC-3'.

Cell Counting Kit 8(CCK-8) assay. A549 and H1299 cells were seeded into 96-well plates at a density of $5 \times 10^{3}$ cells/well and incubated at $37^{\circ} \mathrm{C}$. A total of $10 \mu \mathrm{lCCK}-8$ solution (Dojindo) was added to each well at $0,24,48$ and $72 \mathrm{~h}$. After incubation for $2 \mathrm{~h}$, the optical density value was measured with a microplate reader at a wavelength of $450 \mathrm{~nm}$.

Clonogenic survival assay. NSCLC cells were irradiated with a set of graded doses $(0,2,4,6$ and $8 \mathrm{~Gy})$ and then cultured in 6 -well plates for 14 days. The medium was replaced with 
A

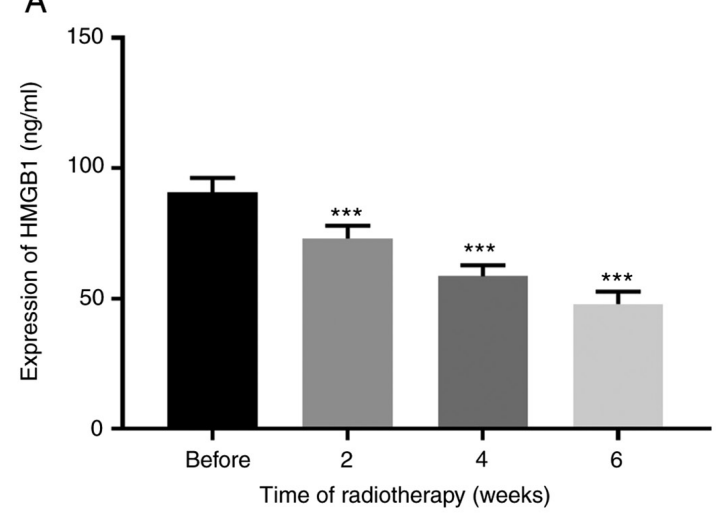

B

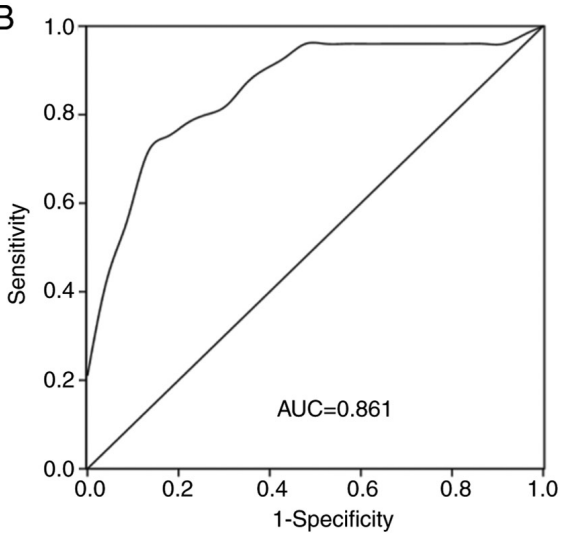

Figure 1. Expression levels and diagnostic value of HMGB1 in the serum of patients with NSCLC. (A) After radiotherapy, the expression levels of HMGB1 were decreased in the serum of patients with NSCLC. ${ }^{* * *} \mathrm{P}<0.001$. (B) Low HMGB1 expression levels distinguished radiosensitive from radioresistant patients, with an AUC of 0.861 ; sensitivity, $74 \%$; specificity, $87 \%$; cutoff value, $91.68 \mathrm{ng} / \mathrm{ml}$. HMGB1, high mobility group box 1; NSCLC, non-small cell lung cancer; AUC, area under the curve.

fresh medium every 3 days. After the formation of cell clones (colonies containing $\geq 50$ cells), the cells were stained with $0.5 \%$ crystal violet solution (Sigma-Aldrich; Merck KGaA) at room temperature for $20 \mathrm{~min}$, and counted under a light microscope (Olympus Corporation).

Transwell invasion assay. Transwell invasion assays were conducted to determine the invasive ability of radioresistant cells, using precoated Matrigel Transwell chambers (BD Bioscience) with $8-\mu \mathrm{m}$ porous membranes (Corning, Inc.). Briefly, A549 cells were seeded into 24-well plates and then exposed to a 2-Gy dose of radiation. After irradiation, $5 \times 10^{4}$ A549 cells resuspended in serum-free medium were seeded into the top chamber, and complete medium was added to the lower chamber as a chemoattractant. After $48 \mathrm{~h}$, invasive cells were fixed with $4 \%$ paraformaldehyde for $10 \mathrm{~min}$ at room temperature, and then stained with $0.1 \%$ crystal violet at room temperature for $20 \mathrm{~min}$. The number of invaded cells was counted in five random independent fields per well using a light microscope (Olympus Corporation).

Luciferase reporter assay. A computer-based algorithm, the TargetScan database (www.targetscan.org), was used to predict potential miRNAs that could target HMGB1. In the present study, miR-107 was searched for in humans following the entry of the HMGB1 gene, after which a luciferase reporter assay was conducted. Briefly, partial sequences of the HMGB1 3'-UTR with wild-type (WT) or mutant (MT) miR-107-binding sites were cloned into the pmirGLO dual-luciferase vector (Promega Corporation) to generate a WT (HMGB1 3'-UTR-WT) or an MT (HMGB1 3'-UTR-MT) reporter plasmid. Then, A549 cells were transiently transfected with the reporter plasmids along with the miR-107 mimics, mimic NC, miR-107 inhibitor or the inhibitor NC using Lipofectamine ${ }^{\circledR} 2000$ (Invitrogen; Thermo Fisher Scientific, Inc.); firefly luciferase activity was evaluated $\sim 8$ h later using a Dual-Luciferase Reporter Assay System (Promega Corporation) and normalized to that of Renilla luciferase.

Western blot analysis. Proteins were extracted from A549 cells using radioimmunoprecipitation assay buffer with protease inhibitor cocktail (both Cell Signaling Technology, Inc.). Protein concentration was determined using a BCA kit (CoWin Biosciences). The proteins (15 $\mu \mathrm{g} /$ lane) were separated using 10\% SDS-PAGE and transferred to nitrocellulose membranes (Amersham; Cytiva). The membranes were subsequently blocked with $5 \%$ skim milk at room temperature for $2 \mathrm{~h}$. Proteins were detected by western blotting using rabbit anti-HMGB1 (1:400; cat. no. ab18256; Abcam), rabbit anti-Toll-like receptor 4 (TLR4; 1:400; cat. no. ab13556; Abcam), rabbit anti-NF-кB p65(1:400; cat. no. ab207297; Abcam), and rabbit anti-GAPDH antibodies (1:1,000; cat. no. ab8245; Abcam) and were incubated at $4^{\circ} \mathrm{C}$ overnight. The membranes were then incubated with the relevant goat anti-rabbit HRP-conjugated secondary antibodies (1:5,000; cat. no. ab7090; Abcam) at room temperature for $1 \mathrm{~h}$, and the protein bands were visualized using ECL reagents (Amersham; Cytiva) and quantified by densitometry (ImageJ software; National Institutes of Health; http://rsbweb.nih.gov).

Statistical analysis. Statistical analyses were performed using GraphPad Prism 5.0 (GraphPad Software, Ltd.) and SPSS 23.0 (IBM Corp.). The data are presented as the mean \pm SD. Patient sample data were non-normally distributed and analyzed by Friedman analysis followed by Nemenyi's post hoc test. Differences between in vitro experimental groups were analyzed by one-way ANOVA followed by Tukey's post hoc test. Receiver operating characteristic (ROC) curve analysis was performed to evaluate the diagnostic value of HMGB1 in identifying radiotherapy-sensitive patients with NSCLC. $\mathrm{P}<0.05$ was considered to indicate a statistically significant difference.

\section{Results}

Serum HMGB1 levels in patients with NSCLC after radiotherapy. The levels of HMGB1 were quantified in the serum of patients with NSCLC who had received radiotherapy. As shown in Fig. 1A, the expression levels of HMGB1 in the serum gradually declined at 2-, 4- and 6-weeks post-radiotherapy compared with those 1 week before radiotherapy $(\mathrm{P}<0.001)$. 

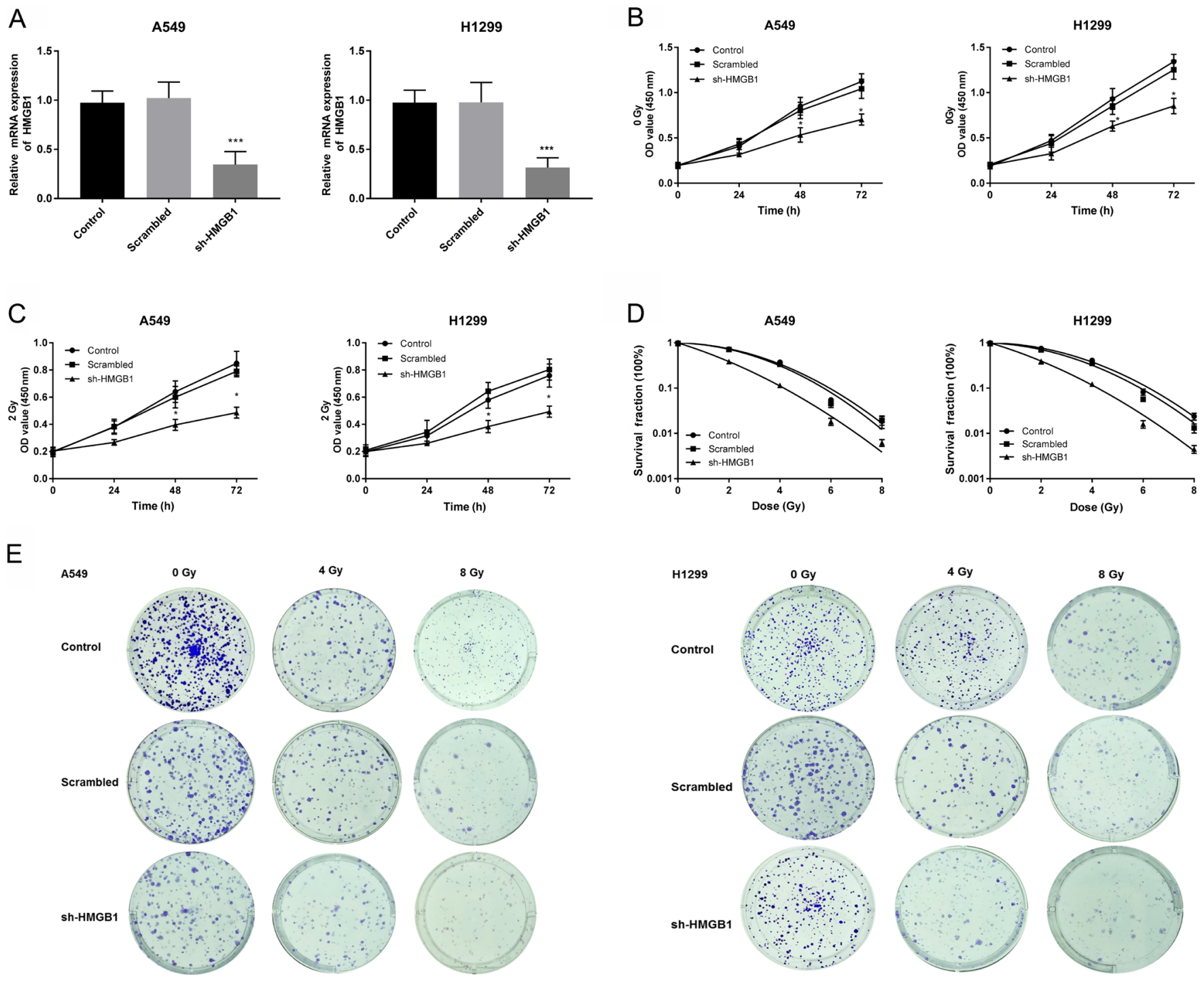

Figure 2. HMGB1-knockdown enhances the radiosensitivity of A549 and H1299 cells. (A) mRNA expression levels of HMGB1 were downregulated in cells transfected with sh-HMGB1, which was determined by reverse transcription-quantitative PCR analysis. (B) HMGB1-knockdown suppressed cellular proliferation compared with the controls. (C) HMGB1-knockdown decreased NSCLC cell proliferation after 2 Gy irradiation. (D) HMGB1-knockdown enhanced the radiosensitivity of NSCLC cells compared with the control. (E) Representative images of Clonogenic survival assay at 0,4 , and 8 Gy. ${ }^{*}<0.05$ and ${ }^{* * *} \mathrm{P}<0.001$ vs. the associated control. HMGB1, high mobility group box 1; NSCLC, non-small cell lung cancer; sh-, short hairpin.

Diagnostic value of HMGB1 for determining the radiosensitivity of patients with NSCLC. Then, the expression levels of HMGB1 before radiotherapy were evaluated for the capacity to distinguish between radiotherapy-sensitive and -resistant patients with NSCLC. As shown in Fig. 1B, ROC curve analysis revealed an area under the curve of 0.861 (sensitivity, 74\%; specificity, $87 \%$; cutoff value, $91.68 \mathrm{ng} / \mathrm{ml}$ ), suggesting that the expression of HMGB1 had the diagnostic accuracy for distinguishing between radiotherapy-sensitive and -resistant patients with NSCLC.

HMGB1-knockdown inhibits the proliferation and increases the radiosensitivity of NSCLC cells in vitro. To investigate the biological function of HMGB1 in NSCLC cell viability, A549 and H1299 cells were transfected with HMGB1 shRNA (sh-HMGB1) to knock down HMGB1expression, and cells treated under different conditions were harvested following transfection and irradiation. RT-qPCR analysis was used to evaluate transfection efficiency. The results indicated that HMGB1 mRNA expression was significantly downregulated in HMGB1-knockdown A549 and H1299 cells compared with that of the control group $(\mathrm{P}<0.001$, Fig. $2 \mathrm{~A})$. The results of the CCK-8 assay revealed that cellular proliferation following sh-HMGB1 transfection was significantly lower than that of the corresponding control cells, both without (Fig. 2B) and with (Fig. 2C) irradiation $(\mathrm{P}<0.05)$. These results suggested that radiation significantly suppressed tumor cell proliferation, and that this inhibitory effect was enhanced when combined with HMGB1-knockdown.

Furthermore, a colony formation assay was conducted to determine the effects of HMGB1 on the radiosensitivity of A549 and H1299 cells. Survival curves were plotted based on the formation of clones and the radiobiological parameters of each group, and the results indicated that compared with the control, HMGB1-knockdown enhanced radiosensitivity compared with the control (Fig. 2D and E). 


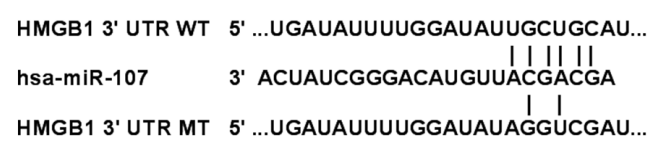

B

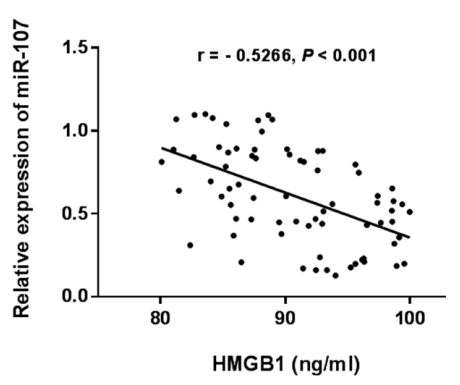

D

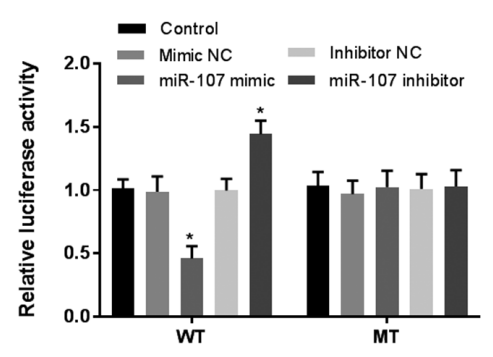

C

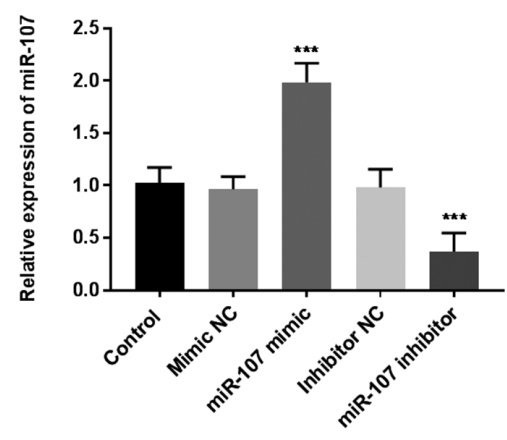

E

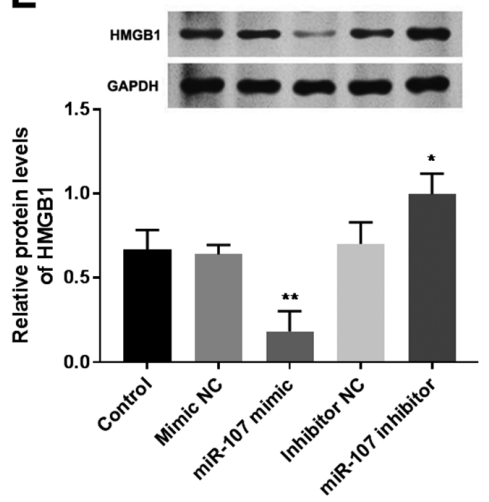

F

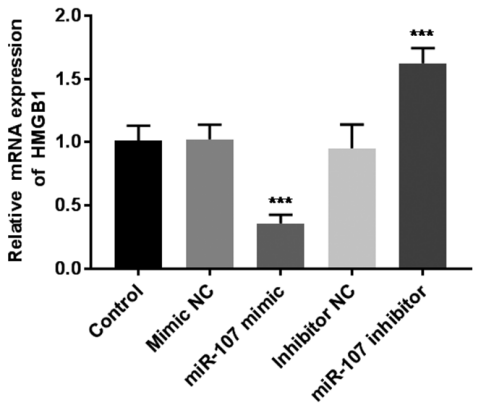

Figure 3. miR-107 negatively regulates HMGB1 expression. (A) Putative miR-107-binding sequences in the HMGB1 3'UTR were predicted using TargetScan. (B) Negative correlation between miR-107 and HMGB1 expression in the serum of patients with NSCLC, determined using Spearman's correlation analysis ( $\mathrm{r}=-0.5266$ and $\mathrm{P}<0.001)$. (C) Transfection efficiency was detected using reverse transcription-quantitative analysis. ${ }^{* * * *} \mathrm{P}<0.001$ vs. the associated control. (D) Luciferase reporters containing WT or MT miR-107 binding sites were transfected into A549 cells along with the miR-107 mimics, mimic NC, miR-107 inhibitor or inhibitor NC. Luciferase activity was evaluated by dual-luciferase reporter assay. "P $<0.05$. (E) miR-107 overexpression decreased the protein levels of HMGB1, while knockdown of miR-107 promoted the HMGB1 protein levels. ${ }^{*} \mathrm{P}<0.05$ and ${ }^{* *} \mathrm{P}<0.01$ vs. the associated control. (F) miR-107 overexpression decreased the mRNA levels of HMGB1, while miR-107-knockdown promoted HMGB1 mRNA expression. ${ }^{* * *} \mathrm{P}<0.001$ vs. the associated control. miR, microRNA; HMGB1, high mobility group box 1; UTR, untranslated region; WT, wild-type; MT, mutant-type; NC, negative control.

These results indicated that transfection with sh-HMGB1 inhibited cellular proliferation with or without irradiation, and increased the radiosensitivity of A549 and H1299 cells in vitro.

HMGB1 is negatively regulated by miR-107 in NSCLC. Previous studies have shown that miRNA dysregulation plays a crucial role in tumor progression by negatively regulating target genes via binding to their 3 '-UTRs. Thus in the present study, bioinformatics analysis was performed using the TargetScan database to predict potential upstream regulators of HMGB1. Among the conserved candidates (miR-107, miR-103 and miR-142-3p), miR-107 was selected as a putative regulator of HMGB1, which was based on the results of a study indicating that miR-107 plays a tumor suppressor role by inhibiting tumor growth and metastasis in NSCLC (19). As shown in Fig. 3A, the HMGB1 3'-UTR contains a putative binding sequence for miR-107. The serum expression levels of miR-107 were also measured by RT-qPCR in patients with NSCLC before radiotherapy, and the correlation between miR-107 expression and HMGB1 levels was assessed. The results showed that HMGB1 level was negatively associated with miR-107 expression in the serum of patients with NSCLC $(r=-0.5266$ and $\mathrm{P}<0.001$, Fig. 3B). The results also indicated that miR-107 mimics promoted the expression of miR-107, while inhibitors suppressed expression ( $\mathrm{P}<0.001$, Fig. 3C). Subsequently, dual-luciferase reporter assays revealed that when A549 cells were transfected with HMGB1 3'-UTR-WT, enhanced expression of miR-107 inhibited, while miR-107-knockdown increased luciferase activity ( $\mathrm{P}<0.05$, Fig. 3D). However, in the cells transfected with HMGB1 3'-UTR-MT, overexpression or downregulation of miR-107 had no significant effect on luciferase activity. Furthermore, the mRNA and protein levels of HMGB1 were determined in A549 cells transfected with miR-107 mimics or inhibitor. The results indicated that miR-107 overexpression decreased the mRNA and protein levels of HMGB1, and that miR-107-knockdown promoted HMGB1 protein $(\mathrm{P}<0.05$, Fig. $3 \mathrm{E})$ and mRNA $(\mathrm{P}<0.001$, Fig. 3F) expression. 
A

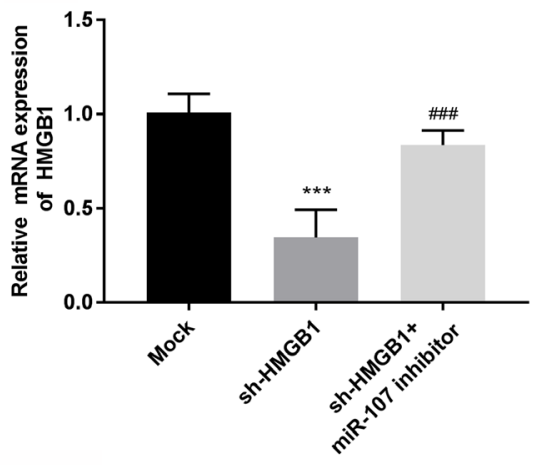

D
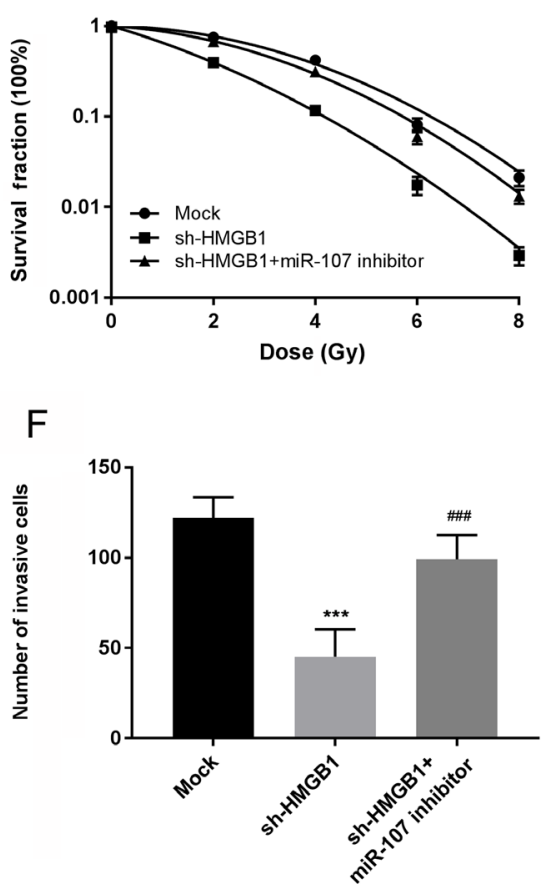

B

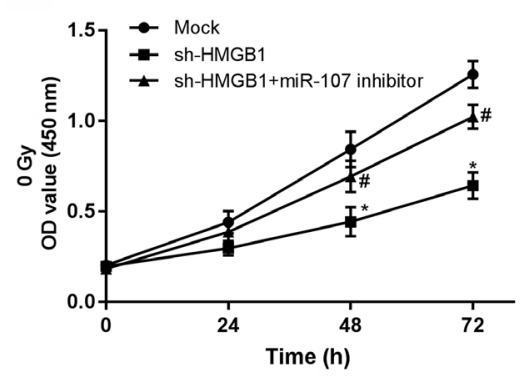

0 Gy $\quad 4$ Gy

E
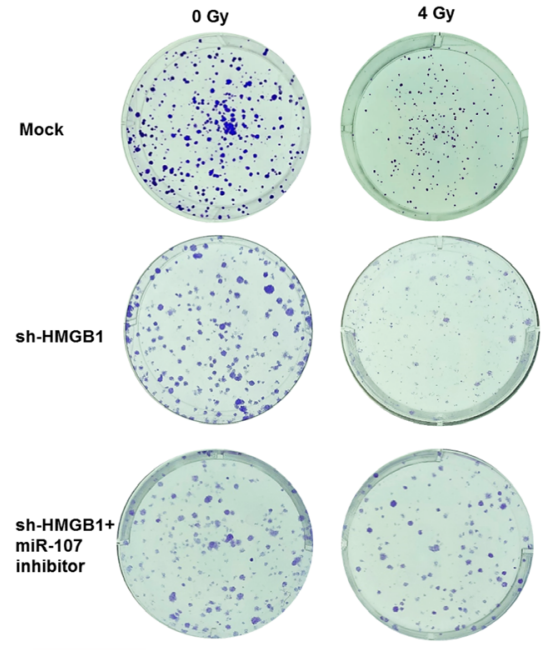

C
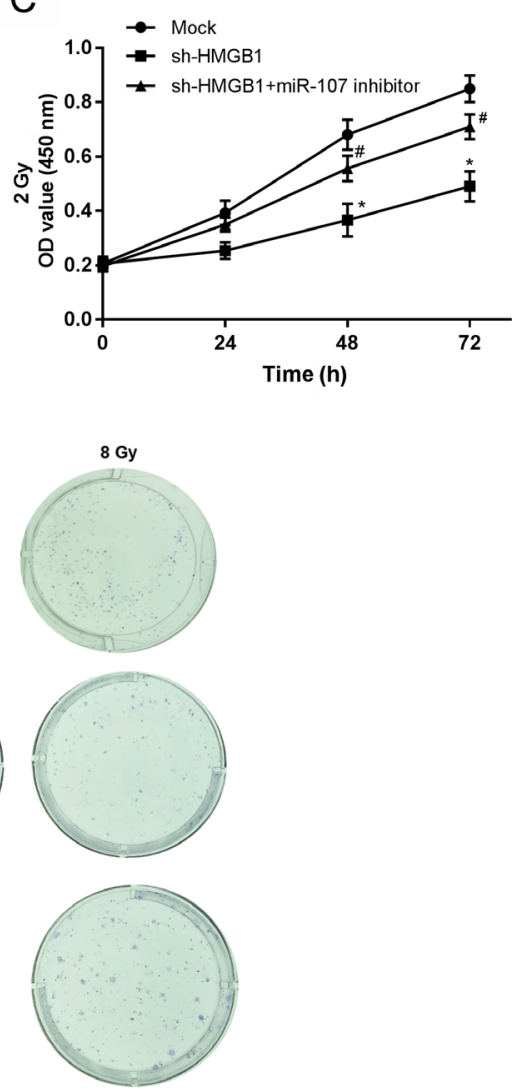

G

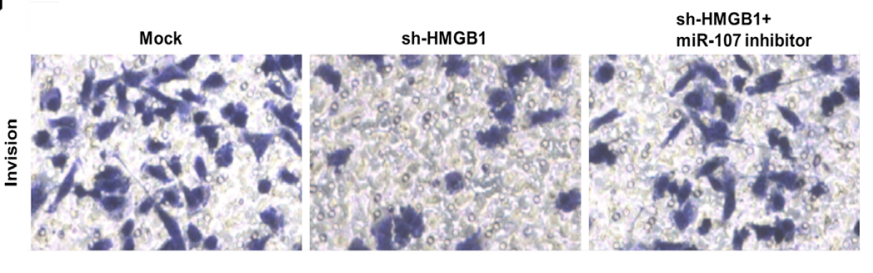

Figure 4. Knockdown of miR-107 expression reversed the increased radiosensitivity of A549 cells induced by HMGB1-knockdown. (A) HMGB1-knockdown reduced the mRNA expression levels of HMGB1, which was reversed by the miR-107 inhibitor in A549 cells. (B) miR-107 inhibitor treatment rescued the decreased proliferative capacity of A549 cells caused by HMGB1-knockdown. (C) miR-107 inhibitor treatment rescued the decreased proliferative capacity caused by HMGB1-knockdown after $2 \mathrm{~Gy}$ of irradiation treatment. (D and E) miR-107-knockdown reversed the reduced survival fractions induced by sh-HMGB1. ${ }^{*} \mathrm{P}<0.05$ and ${ }^{* * *} \mathrm{P}<0.001$ vs. mock; ${ }^{\#} \mathrm{P}<0.05$ and ${ }^{\# \# \#} \mathrm{P}<0.001$ vs. sh-HMGB1. (F and G) Representative Transwell invasion assay images (magnification, $x 200$ ). HMGB1-knockdown suppressed A549 cell invasiveness, and miR-107 downregulation reversed the sh-HMGB1-mediated decrease in invasiveness after irradiation. ${ }^{* * *} \mathrm{P}<0.001$ vs. mock; ${ }^{\# \# \#} \mathrm{P}<0.001$ vs. sh-HMGB1. HMGB1, high mobility group box 1; miR, microRNA; sh-, short hairpin; NC, negative control.

Downregulation of miR-107 relieves the enhanced radiosensitivity of A549 cells caused by sh-HMGB1. To further investigate whether the reduction in HMGB1-enhanced radiosensitivity was regulated by miR-107, A549 cells transfected with sh-HMGB1 or a combination of sh-HMGB1 and the miR-107 inhibitor were treated with or without radiation. The RT-qPCR results showed that sh-HMGB1 downregulated the expression levels of HMGB1, and that knocking down miR-107 reversed the sh-HMGB1-mediated decrease in HMGB1 expression ( $\mathrm{P}<0.001$, Fig. 4A). Knocking down miR-107 with an miR-107 inhibitor reversed the inhibition in cellular proliferation without (Fig. 4B) or with (Fig. 4C) irradiation, and reduced the survival fractions (Fig. 4D and E) induced by sh-HMGB1 $(\mathrm{P}<0.05)$. Furthermore, Transwell invasion assay results showed that HMGB1-knockdown suppressed
A549 cell invasiveness, and that downregulating miR-107 reversed the sh-HMGB1-mediated decrease in the invasion abilities of A549 cells after irradiation $(\mathrm{P}<0.001$, Fig. 4F and G). In summary, these results revealed that the knockdown of HMGB1 enhanced the radiosensitivity of A549 cells regulated by miR-107 expression.

Expression of TLR4/NF- $\kappa$ B signaling pathwayrelated-proteins in A549 cells. Compared with the mock group, HMGB1-knockdown and irradiation downregulated the expression of TLR4/NF- $\mathrm{KB}$ signaling pathway related-proteins $(\mathrm{P}<0.05$, Fig. 5). Compared with the mock-radiation group, the downregulation of HMGB1 enhanced radiation-mediated decreased expression of TLR4/NF- $\mathrm{kB}$ signaling pathway related-proteins in A549 cells $(\mathrm{P}<0.05)$. 

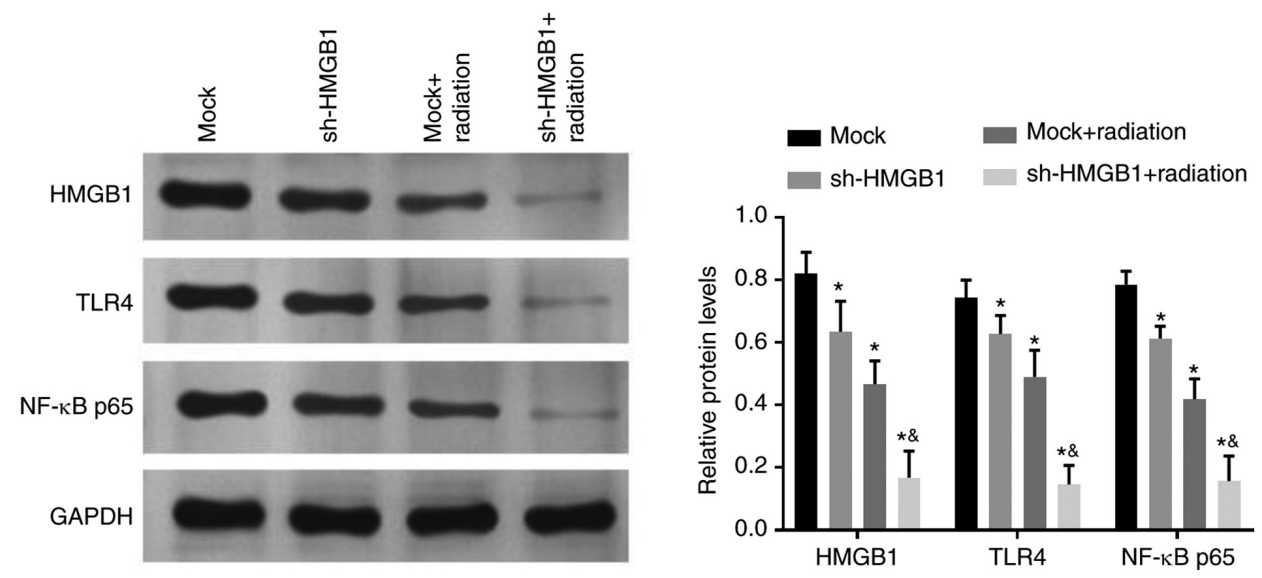

Figure 5. HMGB1-knockdown and irradiation downregulated the expression of TLR4/NF- $\kappa B$ signaling pathway related-proteins. ${ }^{*} \mathrm{P}<0.05$ vs. mock. Downregulation of HMGB1 enhanced the radiation-mediated decreased expression of TLR4/NF- $\mathrm{kB}$ signaling pathway related-proteins. ${ }^{\circledR} \mathrm{P}<0.05$ vs. mock+radiation group. HMGB1, high mobility group box 1; TLR4, Toll-like receptor 4; sh-, short hairpin.

\section{Discussion}

Radiotherapy is an important treatment option for patients with locally advanced NSCLC. However, patients frequently exhibit radioresistance, which in clinical practice, affects treatment efficacy and survival outcome. Thus, increasing radiosensitivity and decreasing radioresistance is crucial for improving patient prognosis. HMGB1 has been reported to be upregulated in various types of cancer, including $\operatorname{NSCLC}(4,22,23)$. However, the effects of HMGB1 on the radiosensitivity of NSCLC remain to be clarified.

HMGB1 is involved in regulating various biological activities, but can also promote tumor development by inducing the formation of tumor neovascularization, and trigger the protective antitumor $\mathrm{T}$ lymphocyte response to inhibit tumor growth (24). In esophageal carcinoma, HMGB1-knockdown decreased the proliferation of tumor cells after irradiation and increased the radiosensitivity of tumor cells (25).

Previous studies have reported that HMGB1 is upregulated in NSCLC and may induce the tumorigenesis and metastasis of lung cancer (26-28). In the present study, HMGB1 expression was notably downregulated in the serum of patients with NSCLC after radiotherapy, suggesting that conventional radiotherapy reduces HMGB1-associated radioresistance, thus inhibiting tumor growth. ROC curve analysis subsequently revealed that the aberrant expression of HMGB1 had a relatively high diagnostic value for distinguishing between radiosensitive and radioresistant patients.

Furthermore, functional assays results demonstrated that HMGB1-knockdown inhibited the proliferation of A549 and H1299 cells, which is consistent with findings in other tumor cell studies $(25,29)$. In addition, irradiation significantly suppressed NSCLC cell proliferation, and this inhibitory effect was enhanced by combining radiotherapy with HMGB1-knockdown. Furthermore, in vitro experimentation also showed that decreased expression of HMGB1 resulted in the heightened radiosensitivity of NSCLC cells. HMGB1-knockdown has also been reported to enhance radiosensitivity in bladder cancer (10) and esophageal squamous cell carcinoma (30), which is consistent with the findings of the present study.
Numerous studies have demonstrated that miRNAs play crucial roles in modulating the progression and tumorigenesis of various cancers by negatively regulating target mRNAs $(31,32)$. In renal carcinoma, the miR-454-3p-induced downregulation of BTG1 enhanced the radiosensitivity of renal carcinoma cells (33). In the present study, only a computer-based algorithm (namely TargetScan) was used to identify a binding association between miR-107 and the HMGB1 3'-UTR. The lack of RNA-RNA binding or protection assays to confirm this association is a limitation to the present study. However, a negative correlation was observed between the levels of HMGB1 and miR-107 in the serum of patients with NSCLC. Subsequent luciferase reporter assay results suggested that miR-107-overexpression inhibited the luciferase activity of A549 cells transfected with the HMGB1 3'-UTR WT sequence, but not with the mutant 3'-UTR sequence. Furthermore, miR-107-overexpression decreased the mRNA and protein levels of HMGB1, while miR-107-knockdown promoted HMGB1 protein and mRNA expression. These results suggest that HMGB1 may be a direct target of miR-107.

Previous studies have also suggested an interaction between miR-107 and HMGB1, including those with human osteoarthritic chondrocytes (34) and breast cancer cells (35). Using A549 cells, the present study reported that knocking down miR-107 reversed the inhibition in cellular proliferation with or without irradiation, and reduced the survival fractions induced by sh-HMGB1. HMGB1-knockdown also suppressed A549 cell invasiveness, which was reversed by downregulating miR-107 after irradiation. These findings suggest that the inhibition in cellular proliferation and invasiveness, and the reduction in survival fractions in NSCLC, associated with HMGB1 downregulation, may be regulated by miR-107.

HMGB1 is an important component of the tumor microenvironment, which leads to immunosuppression and promotes the occurrence and development of tumors (36). TLR4, a member of the Toll-like receptor family, is a crucial component of innate immunity that enhances the function of HMGB1 in tumors by creating a precancerous environment through angiogenesis and inflammation (37). As important survival mechanisms in the response to radiation, various signaling pathways are important for regulating radiosensitivity, 
including the TLK4/NF- $\mathrm{B}$ cascade (38-40). For instance, HMGB1 and the TLR4 signaling pathway were found to be overactive in malignant epithelial ovarian cancer, and to be associated with tumor cell proliferation and invasiveness that may be mediated via NF- $\mathrm{B}$ signaling (41). Moreover, TLR4 and $\mathrm{NF}-\kappa \mathrm{B}$ are closely associated with the radiosensitivity of several cancers, such as esophageal and liver cancer $(42,43)$. In the present study, HMGB1-knockdown and irradiation downregulated the expression of TLR $4 / N F-\kappa B$ signaling pathway related-proteins. Compared with the mock-radiation group, the downregulation of HMGB1 enhanced the radiation-mediated decrease in TLR4/NF- $\kappa \mathrm{B}$ signaling pathway related-protein expression. These findings suggested that HMGB1-knockdown promoted radiosensitivity, which may be regulated by $\mathrm{miR}-107$ via the TLR4/NF- $\mathrm{KB}$ signaling pathway. Accordingly, the present study indicates the potential mechanism by which HMGB1-knockdown results in NSCLC radiosensitivity. In the future, the expression of additonal radiosensitivity-related molecules will be detected to elucidate the detailed mechanism of HMGB1-associated radiosensitivity in NSCLC.

In conclusion, the results of the present study demonstrated that HMGB1 expression was decreased in the serum of patients with NSCLC who had received radiotherapy, and that reduced HMGB1 expression may be a putative diagnostic marker for predicting radiosensitivity in NSCLC. Furthermore, downregulation of HMGB1 enhanced the radiosensitivity of NSCLC cells (that were regulated by miR-107) by mediating the TLR4/NF- $\kappa \mathrm{B}$ signaling pathway. These findings revealed that HMGB1 may be a potential therapeutic target for NSCLC, and that a combination of HMGB1-knockdown and irradiation may be an effective therapeutic strategy for patients with radioresistant NSCLC.

\section{Acknowledgements}

Not applicable.

\section{Funding}

The present study was supported by the Chengde Technology Support Project (grant no. 201701A036).

\section{Availability of data and materials}

The datasets used and/or analyzed during the present study are available from the corresponding author on reasonable request.

\section{Authors' contributions}

$\mathrm{LB}<\mathrm{JZ}, \mathrm{DG}, \mathrm{CL}, \mathrm{WL}$ and $\mathrm{QL}$ made substantial contributions to conception and design of the study, conducting the experiments and interpretation of the data. LB, JZ and DG collected and analyzed the patient data. LB, JZ and CL drafted the manuscript, and WL and QL revised it critically for important intellectual content. LB and QL are responsible for the authenticity of the raw data. All authors approved the final version of the manuscript.

\section{Ethics approval and consent to participate}

The present study was approved by the Ethics Committee of the Affiliated Hospital of Chengde Medical University, and all patients provided written informed consent.

\section{Patient consent for publication}

Not applicable.

\section{Competing interests}

The authors declare that they have no competing interests.

\section{References}

1. Siegel RL, Miller KD and Jemal A: Cancer statistics, 2019. CA Cancer J Clin 69: 7-34, 2019.

2. Larsen JE and Minna JD: Molecular biology of lung cancer: Clinical implications. Clin Chest Med 32: 703-740, 2011.

3. Zhao L, Wang J, Li H, Che J, Ma N and Cao B: Safety and Efficacy of Tianfoshen Oral Liquid in Non-Small Cell Lung Cancer Patients as an Adjuvant Therapy. Evid Based Complement Alternat Med 2019: 1375439, 2019.

4. Cheng P, Ma Y, Gao Z and Duan L: High Mobility Group Box 1 (HMGB1) Predicts Invasion and Poor Prognosis of Glioblastoma Multiforme via Activating AKT Signaling in an Autocrine Pathway. Med Sci Monit 24: 8916-8924, 2018.

5. Hou C, Chen Y, Huang X, Huang Q, Li M and Tan X: miR-19 targets PTEN and mediates high mobility group protein $\mathrm{B} 1(\mathrm{HMGB} 1)$-induced proliferation and migration of human airway smooth muscle cells. PLoS One 14: e0219081, 2019.

6. Yue Y, Zhou T, Gao Y, Zhang Z, Li L, Liu L, Shi W, Su L and Cheng B: High mobility group box 1/toll-like receptor 4/myeloid differentiation factor 88 signaling promotes progression of gastric cancer. Tumour Biol 39: 1010428317694312, 2017.

7. Chen R,Zhu S, Fan XG, Wang H,Lotze MT,Zeh HJ III, Billiar TR, Kang R and Tang D: High mobility group protein B1 controls liver cancer initiation through yes-associated protein-dependent aerobic glycolysis. Hepatology 67: 1823-1841, 2018.

8. Huang CY, Chiang SF, Chen WT, Ke TW, Chen TW, You YS, Lin CY, Chao KSC and Huang CY: HMGB1 promotes ERK-mediated mitochondrial Drp1 phosphorylation for chemoresistance through RAGE in colorectal cancer. Cell Death Dis 9: 1004, 2018.

9. Shi Y and Gong W: Upregulation of miR-129-5p increases the sensitivity to Taxol through inhibiting HMGB1-mediated cell autophagy in breast cancer MCF-7 cells. Braz J Med Biol Res 52: e8657, 2019.

10. Shrivastava S, Mansure JJ, Almajed W, Cury F, Ferbeyre G, Popovic M, Seuntjens J and Kassouf W: The Role of HMGB1 in Radioresistance of Bladder Cancer. Mol Cancer Ther 15: 471-479, 2016.

11. Ma Y, Kang S, Wu X, Han B, Jin Z and Guo Z: Up-regulated HMGB1 in the pleural effusion of non-small cell lung cancer (NSCLC) patients reduces the chemosensitivity of NSCLC cells. Tumori 104: 338-343, 2018

12. Zheng H, Chen JN, Yu X, Jiang P, Yuan L, Shen HS, Zhao LH, Chen PF and Yang M: HMGB1 Enhances Drug Resistance and Promotes In Vivo Tumor Growth of Lung Cancer Cells. DNA Cell Biol 35: 622-627, 2016.

13. Wu X, Wang W, Chen Y, Liu X, Wang J, Qin X, Yuan D, Yu T, Chen G, Mi Y, et al: High Mobility Group Box Protein 1 Serves as a Potential Prognostic Marker of Lung Cancer and Promotes Its Invasion and Metastasis by Matrix Metalloproteinase- 2 in a Nuclear Factor- $\kappa$ B-Dependent Manner. Biomed Res Int 2018: 3453706, 2018.

14. Bai H and Wu S: miR-451: A Novel Biomarker and Potential Therapeutic Target for Cancer. Onco Targets Ther 12: 11069-11082, 2019.

15. Chandan K, Gupta M and Sarwat M: Role of Host and Pathogen-Derived MicroRNAs in Immune Regulation During Infectious and Inflammatory Diseases. Front Immunol 10: 3081, 2020. 
16. Chen G, Yu L, Dong H, Liu Z, Sun Y: MiR-182 enhances radioresistance in non-small cell lung cancer cells by regulating FOXO3. Clin Exp Pharmacol Physiol 46(2):137-143, 2019.

17. Li YH, Xu CL, He CJ, Pu HH, Liu JL and Wang Y: circMTDH.4/miR-630/AEG-1 axis participates in the regulation of proliferation, migration, invasion, chemoresistance, and radioresistance of NSCLC. Mol Carcinog 59: 141-153, 2020.

18. Sun C, Zeng X, Guo H, Wang T, Wei L, Zhang Y, Zhao J, Ma X and Zhang N: MicroRNA-125a-5p modulates radioresistance in LTEP-a2 non-small cell lung cancer cells by targeting SIRT7. Cancer Biomark 27: 39-49, 2020.

19. Xia H, Li Y and Lv X: MicroRNA-107 inhibits tumor growth and metastasis by targeting the BDNF-mediated PI3K/AKT pathway in human non-small lung cancer. Int J Oncol 49: 1325-1333, 2016

20. Zhang Z, Zhang L, Yin ZY, Fan XL, Hu B, Wang LQ and Zhang D: miR-107 regulates cisplatin chemosensitivity of A549 non small cell lung cancer cell line by targeting cyclin dependent kinase 8. Int J Clin Exp Pathol 7: 7236-7241, 2014.

21. Livak KJ and Schmittgen TD: Analysis of relative gene expression data using real-time quantitative PCR and the 2(-Delta Delta C(T)) Method. Methods 25: 402-408, 2001.

22. Amornsupak K, Jamjuntra $P$, Warnnissorn $M$, O-Charoenrat $P$, Sa-Nguanraksa D, Thuwajit P, Eccles SA and Thuwajit C: High $\mathrm{ASMA}^{+}$Fibroblasts and Low Cytoplasmic HMGB1 ${ }^{+}$Breas Cancer Cells Predict Poor Prognosis. Clin Breast Cancer 17: 441-452.e2, 2017.

23. Suren D, Arda Gokay A and Sayiner A: High Mobility Group Box 1 (HMGB1) expression in gastric adenocarcinomas. J BUON 23: 422-427, 2018.

24. Ohmori H, Luo Y, Fujii K, Sasahira T, Shimomoto T, Denda A and Kuniyasu H: Dietary linoleic acid and glucose enhances azoxymethane-induced colon cancer and metastases via the expression of high-mobility group box 1. Pathobiology 77 : 210-217, 2010.

25. Zhang X, Yang X, Zhu S, Li Q and Zou N: Radiosensitization of esophageal carcinoma cells by knockdown of HMGB1 expression. Oncol Rep 41: 1960-1970, 2019.

26. Liu Q, Huo Y, Zheng H, Zhao J, Jia L and Wang P: Ethyl pyruvate suppresses the growth, invasion and migration and induces the apoptosis of non small cell lung cancer cells via the HMGB1/RAGE axis and the NF $\kappa \mathrm{B} / \mathrm{STAT} 3$ pathway. Oncol Rep 42: 817-825, 2019.

27. Wang K, Shan S, Wang S, Gu X, Zhou $X$ and Ren T: HMGB1-containing nucleosome mediates chemotherapy-induced metastasis of human lung cancer. Biochem Biophys Res Commun 500: 758-764, 2018.

28. Wu L and Yang L: The function and mechanism of HMGB1 in lung cancer and its potential therapeutic implications. Oncol Lett 15: 6799-6805, 2018

29. Liu K, Huang J, Ni J, Song D, Ding M, Wang J, Huang $X$ and Li W: MALAT1 promotes osteosarcoma development by regulation of HMGB1 via miR-142-3p and miR-129-5p. Cell Cycle 16 578-587, 2017

30. Di X, He G, Chen H, Zhu C, Qin Q, Yan J, Zhang X and Sun X: High-mobility group box 1 protein modulated proliferation and radioresistance in esophageal squamous cell carcinoma. J Gastroenterol Hepatol 34: 728-735, 2019.
31. Liu R, Chen Y, Shou T, Hu J and Qing C: miRNA-99b-5p targets FZD8 to inhibit non-small cell lung cancer proliferation, migration and invasion. Onco Targets Ther 12: 2615-2621, 2019.

32. Wang L, Lv X, Fu X, Su L, Yang T and Xu P: miR-153 inhibits the resistance of lung cancer to gefitinib via modulating expression of ABCE1. Cancer Biomark 25: 361-369, 2019.

33. Wu X, Ding N, Hu W, He J, Xu S, Pei H, Hua J, Zhou G and Wang J: Down-regulation of BTG1 by miR-454-3p enhances cellular radiosensitivity in renal carcinoma cells. Radiat Oncol 9: 179, 2014.

34. Lin SS, Yuan LJ, Niu CC, Tu YK, Yang CY and Ueng SWN: Hyperbaric oxygen inhibits the HMGB1/RAGE signaling pathway by upregulating mir-107 expression in human osteoarthritic chondrocytes. Osteoarthritis Cartilage 27: 1372-1381, 2019.

35. Ai H, Zhou W, Wang Z, Qiong G, Chen Z and Deng S: MicroRNAs-107 inhibited autophagy, proliferation, and migration of breast cancer cells by targeting HMGB1. J Cell Biochem. Dec 2, 2018 (Epub ahead of print). doi: 10.1002/jcb.28157.

36. Chen G, Ward MF, Sama AE and Wang H: Extracellular HMGB1 as a proinflammatory cytokine. J Interferon Cytokine Res 24 329-333, 2004.

37. Li Z, Block MS, Vierkant RA, Fogarty ZC, Winham SJ, Visscher DW, Kalli KR, Wang C and Goode EL: The inflammatory microenvironment in epithelial ovarian cancer: A role for TLR4 and MyD88 and related proteins. Tumour Biol 37: 13279-13286, 2016.

38. Bai M, Ma X, Li X, Wang X, Mei Q, Li X, Wu Z and Han W: The Accomplices of NF- $\kappa$ B Lead to Radioresistance. Curr Protein Pept Sci 16: 279-294, 2015.

39. Jung CH, Han AR, Chung HJ, Ha IH and Um HD: Linarin inhibits radiation-induced cancer invasion by downregulating MMP-9 expression via the suppression of NF- $\mathrm{B}$ activation in human non-small-cell lung cancer A549. Nat Prod Res 33: 3582-3586, 2019

40. Liu YC, Chiang IT, Hsu FT and Hwang JJ: Using NF- $\kappa$ B as a molecular target for theranostics in radiation oncology research. Expert Rev Mol Diagn 12: 139-146, 2012.

41. Jiang C, Qu X, Ke H, Gong W, Chen R, Yang W and Cheng Z: Association between the HMGB1/TLR4 signaling pathway and the clinicopathological features of ovarian cancer. Mol Med Rep 18: 3093-3098, 2018.

42. Tang H, Wang F, Zhou XF, Zhou J, Chen L, Luo JD and Xu EC: Relationship between TLR4 and NF- $\kappa \mathrm{B}$ p 65 protein expressions and clinical radiosensitivity of patients with esophageal squamous cell carcinoma. Pak J Med Sci 30: 982-985, 2014.

43. Zhi-Feng W, Le-Yuan Z, Xiao-Hui Z, Ya-Bo G, Jian-Ying Z, Yong $\mathrm{H}$ and Zhao-Chong Z: TLR4-dependent immune response promotes radiation-induced liver disease by changing the liver tissue interstitial microenvironment during liver cancer radiotherapy. Radiat Res 182: 674-682, 2014.

This work is licensed under a Creative Commons Attribution-NonCommercial-NoDerivatives 4.0 International (CC BY-NC-ND 4.0) License. 